\title{
Cardioprotection by direct factor Xa inhibition in angiotensin II overexpression
}

\author{
Masaki Mogi ${ }^{1}$
}

Received: 7 July 2021 / Accepted: 13 July 2021 / Published online: 4 August 2021

(C) The Japanese Society of Hypertension 2021

Direct factor Xa (FXa) inhibitors have been used as active oral anticoagulant agents to prevent stroke and deep vein thrombosis. Recently, the anti-inflammatory effects of FXa inhibitors have been expected to reduce the risk of future cardiovascular events via its effect on various signaling pathways through activation of protease-activated receptors (PARs) and non-PARs [1]. PARs are seven-transmembrane domain receptors that are activated by the specific proteolytic cleavage of the N-terminal extracellular domain by endogenous serine proteases, such as thrombin (which acts on PAR-1, -3, and -4) and trypsin, tryptase, factor VIIa, and FXa (which act on PAR-2), and the released N-terminal tail binds the receptor itself and induces intracellular signaling [2].

The present study by Narita et al. demonstrates that a direct FXa inhibitor, rivaroxaban (Riv), has a protective effect against cardiac hypertrophy and fibrosis development via PAR-2 inhibition in angiotensin (Ang) II-overexpressing (Ren-Tg) mice. Treatment with Riv reduced blood pressure and cardiac hypertrophy and fibrosis, with reduced proinflammatory and profibrotic cytokine expression. This group performed experiments with FSLLRY, a PAR-2 antagonist, and the results suggest a similar effect of Riv and FSLLRY on signaling pathways, such as the phosphorylationactivated Erk pathway. Narita et al. had previously reported the protective effects of Riv against renal damage through the inhibition of PAR-2 signaling-mediated inflammation in Ren-2 mice [3]. Thus, the present study is a subseries report of their research focusing on PAR signaling in Ren-2 mice. The effects of Riv on cardiovascular disease have also been recently reported by others. Bode et al. demonstrated that early administration of Riv preserves cardiac function in left anterior descending artery-ligated

Masaki Mogi

mmogi@m.ehime-u.ac.jp

1 Department of Pharmacology, Ehime University, Graduate School of Medicine, Shitsukawa, Tohon, Ehime, Japan models established with PAR-2-deficient mice [4]. Using a myocardial infarction model, Nakanishi et al. also reported cardioprotective effects of Riv via downregulation of the PAR-2 pathway [5]. Moreover, a relationship between Ang II and PAR signaling in PAR-1-deficient mice was reported by Antoniak et al. [6]. PAR-1-knockout mice treated with a subpressor dose of Ang II for 4 weeks failed to cause vascular remodeling of the aorta but did induce cardiac hypertrophy, in contrast to the effect on wild-type mice, indicating that PAR-1 plays a significant role in "vascular" remodeling induced by Ang II without raising blood pressure. Narita et al. also previously demonstrated cardioprotective effects of PAR-1 inhibition by SCH79797, a PAR-1 antagonist, on cardiac hypertrophy and fibrosis in Ren-2 mice [7]. They also showed that the FXa-induced enhancement of hypertrophic signaling in isolated cardiac fibroblasts was abolished by SCH79797, indicating that both PAR-1 and PAR-2 are activated in association with FXa in Ren-2 mice. Although Rev mainly affects PAR-2 signaling, FXa inhibition may also reduce PAR-1 signaling. Therefore, continuous activation of the renin-angiotensin system (RAS) may synergistically or additively affect PAR signaling, resulting in the induction of cardiovascular dysfunction.

The present study shows that treatment with Riv induces blood pressure reduction in Ren-2 mice. PAR-2 activation induces vasorelaxation associated with endothelial activation partially through the release of nitric oxide [8]. McGuire et al. reported that baseline blood pressure is higher in PAR-2-deficient mice, but a lower blood pressure trend was observed in PAR-2-deficient mice fed a high salt diet and treated with Ang II but not in wild-type mice [9]. As described in the Discussion, the underlying mechanism of the SBP-lowering effect of Riv is unclear. The inhibitory effects of Riv on PAR-2-induced vascular inflammation may be involved, but whether there is a relationship between PAR-2 signaling and the Ang II type 1 receptor $\left(\mathrm{AT}_{1} \mathrm{R}\right)$ in blood pressure regulation has not been elucidated. If Riv acts independently in RAS regulation of blood 
Fig. 1 Correlation between PARs and RAS in cardiac hypertrophy and fibrosis and blood pressure elevation. Ang II angiotensin II, AT1R angiotensin II type 1 receptor, ARB AT1R blocker, FXa factor $\mathrm{Xa}$, PAR protease-activated receptor, p-ERK phosphorylated extracellular signal-regulated kinase, RAS renin-angiotensin system, Riv rivaroxaban

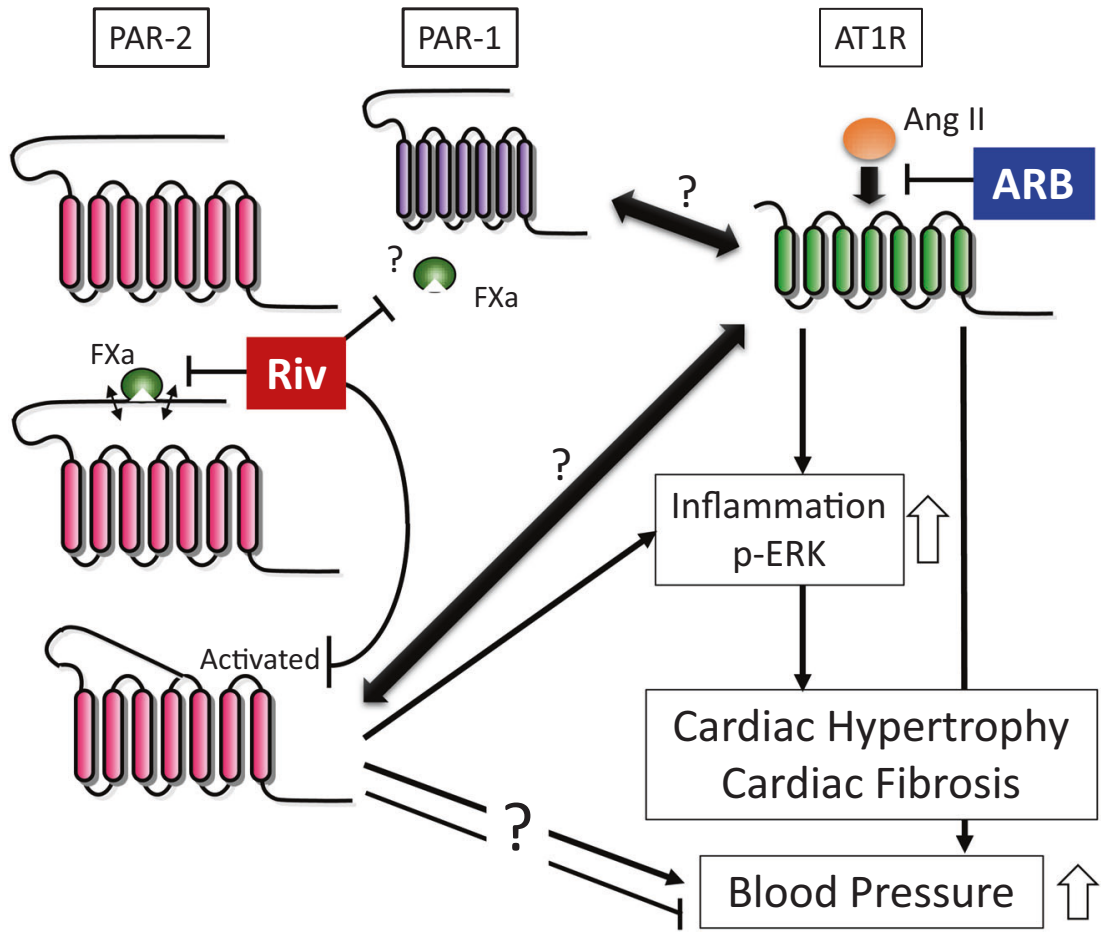

pressure, then combined effects of an $\mathrm{AT}_{1} \mathrm{R}$ blocker (ARB) and Riv can be expected. Poststroke patients and patients with atrial fibrillation are commonly treated with both antihypertensive and anticoagulation drugs. Therefore, to prove that cotreatment with RAS blockade and direct FXa inhibition has additive or synergistic effects, basic animal studies and clinical research focusing on blood pressure and organ damage in patients treated with a combination treatment of $\mathrm{ARB}$ and Riv are needed (Fig. 1).

\section{Compliance with Ethical Standards}

Conflict of interest The author declares no competing interests.

Publisher's note Springer Nature remains neutral with regard to jurisdictional claims in published maps and institutional affiliations.

\section{References}

1. Ebrahimi S, Rezaei S, Seiri P, Ryzhikov M, Hashemy SI, Hassanian SM. Factor Xa signaling contributes to the pathogenesis of inflammatory diseases. J Cell Physiol. 2017;232:1966-70.
2. Macfarlane SR, Seatter MJ, Kanke T, Hunter GD, Plevin R Proteinase-activated receptors. Pharm Rev. 2001;53:245-82.

3. Ichikawa H, Shimada M, Narita M, Narita I, Kimura Y, Tanaka M, et al. Rivaroxaban, a direct factor Xa inhibitor, ameliorates hypertensive renal damage through inhibition of the inflammatory response mediated by protease-activated receptor pathway. J Am Heart Assoc. 2019;8:e012195.

4. Bode MF, Auriemma AC, Grover SP, Hisada Y, Rennie A, Bode $\mathrm{WD}$, et al. The factor $\mathrm{Xa}$ inhibitor rivaroxaban reduces cardiac dysfunction in a mouse model of myocardial infarction. Thromb Res. 2018;167:128-34.

5. Nakanishi N, Kaikita K, Ishii M, Oimatsu Y, Mitsuse T, Ito M, et al. Cardioprotective effects of rivaroxaban on cardiac remodeling after experimental myocardial infarction in mice. Circ Rep. 2020;2:158-66.

6. Antoniak S, Cardenas JC, Buczek LJ, Church FC, Mackman N, Pawlinski R. Protease-activated receptor 1 contributes to angiotensin II-induced cardiovascular remodeling and inflammation. Cardiology. 2017;136:258-68.

7. Yokono Y, Hanada K, Narita M, Tatara Y, Kawamura Y, Miura N, et al. Blockade of PAR-1 signaling attenuates cardiac hypertrophy and fibrosis in renin-overexpressing hypertensive mice. J Am Heart Assoc. 2020;9:e015616.

8. Cicala C. Protease activated receptor 2 and the cardiovascular system. Br J Pharm. 2002;135:14-20.

9. McGuire JJ, Van Vliet BN, Halfyard SJ. Blood pressures, heart rate and locomotor activity during salt loading and angiotensin II infusion in protease-activated receptor 2 (PAR2) knockout mice. BMC Physiol. 2008;8:20. 